\title{
Public Perception and Potential Acceptance of COVID-19 Vaccine in India
}

\author{
Bibhabasu Das. ${ }^{1 *}$ Padhye A. ${ }^{2}$ \\ DOI: https://doi.org/10.17511/ijphr.2021.i02.03 \\ 1* Bibhabasu Das, Department of Mechanical Engineering, Indian Institute of Technology Madras, Chennai, Tamil Nadu, India. \\ 2 Apurva Padhye, Department of Physics, Indian Institute of Technology Madras, Chennai, Tamil Nadu, India.
}

The COVID-19 pandemic, caused by Severe acute respiratory syndrome coronavirus 2 (SARS-CoV-2) virus, has led to a substantial loss of human life and the global economy, and presents an unprecedented collapse of the public health systems worldwide. The sped-up advancement of the COVID-19 vaccine is an important development. Data from the early trials suggest that the vaccine is safe as well as effective. However, the acceptance of the COVID-19 vaccine among the public depends on various socio-demographic factors. The primary aim of the study is to get a deeper understanding and analysis of the public's perception, information and sentiment towards the COVID-19 vaccine in India.

Keywords: COVID-19, Vaccine Hesitancy, Vaccine Acceptance

\section{Corresponding Author}

Bibhabasu Das, Department of Mechanical Engineering, Indian Institute of Technology Madras, Chennai, Tamil Nadu, India.

Email: me17b134@smail.iitm.ac.in

\section{How to Cite this Article}

Das B, Padhye A. Public Perception and Potential Acceptance of COVID-19 Vaccine in India. Public Health Rev Int J Public Health Res. 2021;8(2):23-31. Available From https://publichealth.medresearch.in/index.php/ijphr/ article/view/156
To Browse

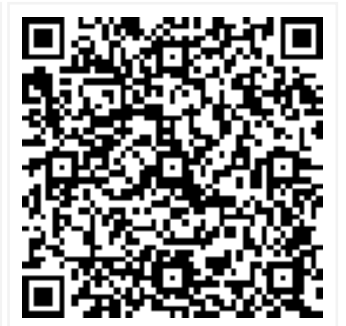

Manuscript Received 2021-04-04

Conflict of Interest No
Review Round 1 2021-04-14

Funding $\mathrm{Nil}$
Review Round 2

2021-04-24

Ethical Approval Yes
Review Round 3

Accepted 2021-04-30

Plagiarism X-checker $9 \%$ 


\section{Introduction}

Coronavirus disease 2019 (COVID-19) is a large virus family that can infect both animals and humans. Several coronaviruses have been identified in humans, and they can cause serious respiratory infections ranging from the common cold to more serious diseases like extreme acute respiratory syndrome (SARS), Middle East respiratory syndrome (MERS), and coronavirus disease 2019. (COVID-19). SARS-CoV-2 has spread through the borders of approximately 200 countries, affecting about 150 million people and killing approximately 3 million people.

Owing to their poor economic status and depleting resources available in their countries, the SARSCoV-2 (COVID 19) epidemic has had a hugely negative effect on many countries' public health systems. Since there are no clear universal antiviral drugs for SARS-CoV-2, the World Health Organization (WHO) recommendations are currently being followed in preventing further outbreaks, early detection of those who have been infected, and developing a comprehensive and reliable treatment. However, over the last few months, several researchers from around the world have assessed the safety, efficacy, and effectiveness of many repurposed drugs for the treatment of COVID19. Among the therapeutic vaccines that have been studied, a few of them have shown promise in reducing and improving overall mortality in COVID19 patients.

We can see how maintaining social distance norms and using secure personal protective equipment with national lockdowns over a prolonged period is improbable. As a result, the only way to stop the pandemic from spreading further is to create a scientifically safe and widely available COVID 19 vaccine and make it available to the general public around the world. [1]

This research provides insight into the key variables and obstacles that contribute to low vaccine acceptance rates, and it can help us prepare the required actions and steps to raise public awareness and strengthen public confidence in the vaccines' protection, efficacy, and benefits. These variables frequently include socio-demographic and economic factors, as well as old norms and anti-vaccination attitudes in the battle against COVID 19. This will enable us to monitor and slow the pandemic's spread, as well as prevent the pandemic's negative effects from worsening.
Evaluation of public opinion and the cause of vaccine hesitancy will aid in the development of improved and more efficient communication awareness campaigns in different communities to identify the various norms that the public perceives to have for the vaccine and to foster confidence in health and public authorities.

\section{Vaccine Hesitancy}

A vaccine is considered the most awaited intervention, and hundreds of global R\&D institutions are working on it at a rapid pace. However, there is no information on public perception of COVID-19 vaccine uptake. [2] Several studies have identified several factors that influence vaccine acceptance when a new vaccine is introduced. [3,4,5] These include concerns about the vaccine's safety and effectiveness, negative health effects, misunderstandings about the need for vaccination, a lack of confidence in the health system, and a lack of community awareness about vaccine-preventable diseases. Misinformation that leads to vaccine hesitancy could jeopardise public health in the face of the current crisis. $[6,7]$.

Vaccine hesitancy among the general public is one of the oldest phenomena that has posed a threat to global health, especially during outbreaks of infectious diseases (e.g., measles, H1N1, and pertussis). The rapid production of COVID-19 vaccines that are both effective and safe is truly remarkable. COVID-19 vaccine hesitancy, on the other hand, has frequently been the primary limiting measure in containing the current pandemic's negative health and socioeconomic consequences.

To curb and flatten the covid 19 global case curve, we must understand that high vaccine coverage at the community level is necessary. Vaccine hesitancy affects both the person who refuses to take the vaccine and the entire population, posing a significant challenge to reaching the threshold for conferring COVID-19 herd immunity. We need to understand and boost population immunity, which is highly dependent on the COVID-19's basic reproductive number, to limit the spread of SARSCoV-2.

According to the most recent COVID-19 figures, 6075 percent of the community population will need to be resistant and vaccinated to stop and minimise COVID 19 virus transmission and curb the spread at the community level. 
But, the level of vaccine hesitancy and acceptances in the public population can be a major pivotal factor that could obstruct the successful control of the ongoing pandemic caused by COVID-19. [2] Vaccination hesitancy is often triggered by public misconceptions and barriers about the vaccines' safety, efficacy, and side effects. The public's understanding and acceptance of it are influenced by a variety of factors, including the community's behaviour and literacy, as well as their geographic location. Furthermore, several important factors influence vaccine acceptance, including the seriousness of the disease, previous vaccination experience in their population, disbelief in local healthcare systems, route of administration, socioeconomic and literacy status of individuals, faith in doctors'/physicians' recommendations, and vaccine cost-accessibility. The 2009 H1N1 pandemic, which had shown unsatisfactory results and had a very poor vaccine acceptance rate in the range of 17 to 67 percent across different countries, was one of the first instances of public assistance regarding vaccines against emerging pandemics. COVID-19, on the other hand, has a higher morbidity and mortality rate due to its pandemic spread, prompting the creation of vaccine candidates for this virus. As a result, gaining a better understanding of public opinion and common barriers to vaccine acceptance will help us increase overall coverage rates in the general population. [8] Vaccine hesitancy may be exacerbated by rumours and conspiracy theories. Monitoring COVID-19 vaccine candidate data on the internet will help track vaccine misinformation in real-time and mitigate its effect. A cross-sectional study examined COVID-19 vaccine myths and conspiracy theories that were circulating on the online platforms, understood their background, and then looked at interventions to deal with the misinformation and increase vaccine acceptance. Between December 31, 2019 and November 30,2020 , a multi-disciplinary team was created June 2020 to study and compile online rumours and conspiracy theories. There were 637 COVID-19 vaccine-related items included in the study: From 52 nations, 91 percent were rumours and $9 \%$ were conspiracy theories. Sixty-six percent of the 578 rumours were about vaccine production, availability, and access, twenty percent were about morbidity and mortality, eight percent were about safety, effectiveness, and acceptance, and the rest were about other topics. 5 percent (30) of the 637 items were right, 83 percent (528/637) were incorrect, $10 \%(66 / 637)$ were misleading, and $2 \%$
(13/637) were exaggerated. A large amount of COVID-19 vaccine misinformation circulating may undermine the vaccine candidates' universal rollout. Traditional risk communication and community involvement approaches should be investigated to monitor and fact-check misinformation as a way to immunise people against misinformation and thereby prevent future vaccination programme disturbances, according to the report.

These results should be taken into account by policymakers when developing risk communication and community involvement plans to resolve these issues with evidence-based data. Topic modelling, artificial intelligence, and machine learning technology also can monitor and analyse a large amount of media data in real-time; however, these technologies can be prohibitively costly for low- and middle-income countries. [9]

\section{COVID-19 Vaccines Phase Development}

The vaccine development phase includes a fourstage clinical testing process that aims to better understand and enhance the vaccine's safety, efficiency, and efficacy against COVID 19. When a candidate vaccine is ready for human clinical trials, it goes through Phase 1 trials, which are designed to assess the vaccine's efficacy. It typically involves 20-100 healthy volunteers and is used to assess dosages, side effects, and to learn more about how the vaccine works.

Phase 2 studies delve further into the safety of the treatment and begin to understand and investigate its effectiveness in a wider group of volunteers (around a few hundred volunteers). Phase 3 trials, where only a small percentage of vaccines make it to, are conducted on a wider group of participants, involving thousands or tens of thousands of individuals, to validate and measure the vaccine's efficacy as well as identify any rarer side effects.' It also aids in the understanding of post-vaccination symptoms.

After it has been approved by a national regulatory body, phase 4 trials are carried out, which entails further testing in a large population for a longer period as a method of post-marketing surveillance (pharmacovigilance). The World Health Organization chooses the applicants for clinical trials at different times (WHO). [10] 


\section{Different types of COVID-19 Vaccine}

The primary goal of the Covid 19 vaccines is to help our bodies develop immunity to the virus that causes COVID-19 without causing us to get sick. There are many types of vaccines, each with its genetic mechanism for developing and enhancing our defences against the COVID 19 Virus. But one thing remains constant: the body must develop a mechanism to aid in the production of primary antibodies, which are T-lymphocytes and Blymphocytes that know how to react to the virus effectively in the future. However, the process of building an immunity system against the deadly COVID 19 virus takes a few weeks post-vaccination as it takes time for our body to produce these antibodies. Therefore, a person can be infected with the virus during this time post-vaccination. [11] As of January 2021, nine separate technology systems are being researched and developed to produce an efficient and reliable COVID 19 vaccine, with the technology of several candidates remaining unknown. Currently, there are three major types of vaccines in development (in Phase 3/4: large-scale clinical trials) and approved for production by WHOrecognized national authorities.' The following is a detailed explanation of how each of these work:

- mRNA Vaccines: They include material from the virus that induces COVID-19, which instructs and assists our cells in producing a harmless virus-specific protein.' Once our cells have figured out how to reproduce these proteins, the genetic material in the vaccines is destroyed. Our bodies are then taught to recognise that these proteins should be destroyed and to aid in the development of Tand B-lymphocytes, which will aid our bodies in recognising and fighting the SARS CoV 2 virus if we become infected in the future. [11]

- COVID 19 protein subunit vaccines: Instead of the whole virus, these vaccines contain the virus's harmless protein fragments. It recognises the presence of the protein in the same way that mRNA does, and it aids in the development of T-lymphocytes and antibodies that are needed to create immunity against the SARS CoV 2 virus.[11]

- Vector vaccines: A modified genetic material of a virus that is slightly different from SARS CoV 2 is used to create one of the most powerful and stable vaccines.
The primary function of this genetic material is to create immunity by instructing the development of a protein specific to the COVID19 virus. Once within the body, the genetic material instructs the cells to produce a special protein for SARS CoV 2, which aids in the formation of T-lymphocytes and B-lymphocytes in our bodies. These proteins help our immune system in recognising the virus in the future and remembering how to combat it. [11]

\section{COVID-19 Vaccines in India}

On 16 January 2021, the Indian government started its administration of the COVID 19 vaccination campaign across the nation. As of 4 May 2021, the Indian government and Serum Institute of India and Bharat Biotech have successfully administered $160,494,188$ currently approved vaccine doses overall, including the first and second doses. The two vaccines in India that have received the green light for emergency use have been Covishield (a version of the viral vector Oxford-AstraZeneca vaccine developed by the Serum Institute of India) and Covaxin (Developed by Bharat Biotech). With the need of improving the current vaccination coverage across different states due to insufficient vaccine supply, Sputnik $\mathrm{V}$ was approved by the Indian government as a third vaccine in April 2021. The expected deployment is to start in late May 2021. [12]

India is known as the world's vaccine production centre, accounting for $60 \%$ of global vaccine availability. The country can produce more than 3 billion doses of coronavirus disease 2019 (COVID19) vaccine per year. Low-income countries that cannot afford costly vaccines will benefit from the country's ability to manufacture low-cost COVID-19 vaccines. For the production and manufacturing of COVID-19 vaccines, the majority of Indian vaccine manufacturers have signed exclusive licence agreements with international collaborators. Bharat Biotech produced and manufactured Covaxin (BBV152), India's first indigenous COVID-19 inactivated vaccine, in partnership with the Indian Council of Medical Research (ICMR) and the National Institute of Virology (NIV).

Covaxin will effectively neutralise the recently emerging B 1.1.7 SARS-CoV-2 variant, according to recent findings (UK variant). Bharat Biotech has also begun a phase 1 trial (NCT04751682) for COVID-19 using a single-dose adenovirus vectored intranasal vaccine (BBV154). 
Covishield is the Indian version of Oxford University and AstraZeneca's replication-deficient adenoviral vector vaccine (AZD1222, previously called ChAdOx1 nCoV-19 vaccine). The Serum Institute of India (SII), the world's largest vaccine producer and one of the world's leading vaccine exporters, produces it. COVI-VAC, a live-attenuated intranasal vaccine against COVID-19, was developed in collaboration with SII and Codagenix. According to the Department of Biotechnology (DBT), India can manufacture 70-100 million doses of Covishield vaccine per month. In comparison, the indigenously developed Covaxin is limited to 150 million doses per year. [12]

India began a nationwide vaccination campaign in early 2021, and as of April 8, 2021, it had vaccinated 87.1 million people. Covaxin and Covishield vaccines are currently being used in India's vaccination campaign since they are the only vaccines that have earned clearance from the Central Drugs and Standards Committee (restricted use in emergencies). [12].

\section{A General Overview and Factors Affecting the Vaccine Acceptance in India}

These are results from a study conducted with 351 participants using an online questionnaire in October 2020. It was aimed at analyzing the beliefs regarding the COVID-19 vaccine and the barriers leading to vaccine hesitancy among the general population of India. The study population was mainly ( $85 \%)$ highly educated. A significant section of the respondents $(>75 \%)$ were from the age group 19-29 years. [13]

\section{Opinion on safety and effectiveness of the COVID-19 vaccine}
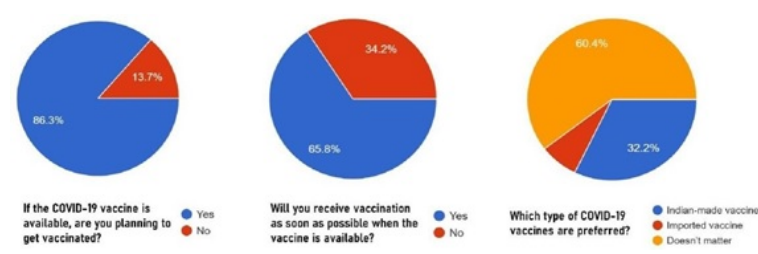

Figure 1 Response of participants regarding their beliefs towards safety and effectiveness of COVID19 vaccination. Image from Sharun et al. COVID-19 Vaccine Acceptance: Beliefs and Barriers Associated with Vaccination Among the General Population in India. J Experiment Bio Agri Sci. 2020; 8:S210-8 [13]
$55 \%$ of the participants believed that COVID-19 vaccination would be safe, but only $46.2 \%$ believed it would be effective. $38.5 \%$ reported uncertainty regarding safety and $45 \%$ regarding the effectiveness of the vaccine. The majority (68.1\%) agreed that vaccination is the best way to avoid severe threats from the COVID-19 virus. [13]

\section{COVID-19 vaccine acceptance}
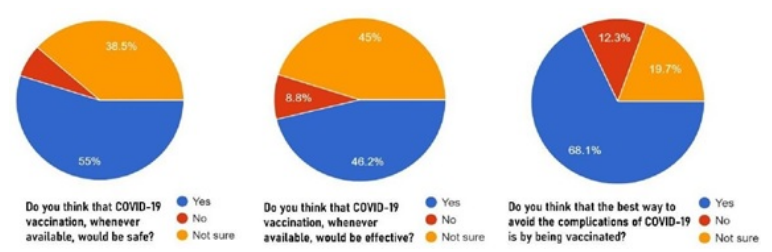

Figure 2 Response of participants regarding the acceptance of COVID-19 vaccine. Image from Sharun et al. COVID-19 Vaccine Acceptance: Beliefs and Barriers Associated with Vaccination Among the General Population in India. J Experiment Bio Agri Sci. 2020; 8:S210-8 11 [13]

The vaccine acceptance was found to be good, with $86.3 \%$ of participants planning to get vaccinated whenever the vaccine becomes available, whereas $13.7 \%$ of the participants did not want to get vaccinated. 'However, the percentage of participants ready to get themselves vaccinated as soon as a vaccine is ready and available was only $65.8 \%$. $32.2 \%$ of the participants gave preference to an Indian vaccine, while $7.5 \%$ preferred an imported one. The majority $(60.4 \%)$ reported that they do not care about the country of origin of the vaccine. We can see that more than three-fourths of the respondents were positive about receiving the vaccination; whereas, only two-third were intending to accept it as soon as possible when the vaccine became available. [13]

\section{Barriers associated with the COVID-19 vaccine}

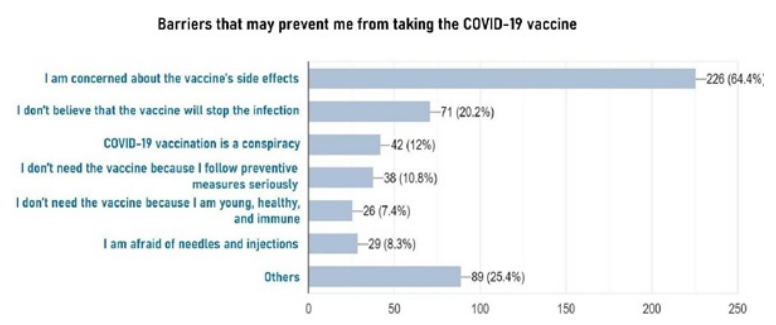

Figure 3 Response of participants regarding the barriers associated with COVID-19 vaccination. Image from Sharun et al. COVID-19 Vaccine Acceptance: Beliefs and Barriers Associated with Vaccination Among the General Population in India. 


\section{J Experiment Bio Agri Sci. 2020;8:S210-8}

For most (64.4\%), the main concern was regarding the side effects of the COVID-19 vaccine. $20.2 \%$ of the respondents were not confident about the efficacy of vaccination, while $12 \%$ believed that the COVID-19 vaccine is some conspiracy. A significant number $(25.4 \%)$ had other reasons preventing them from getting themselves vaccinated. [13]

\section{Factors that can improve COVID-19 vaccine acceptance}

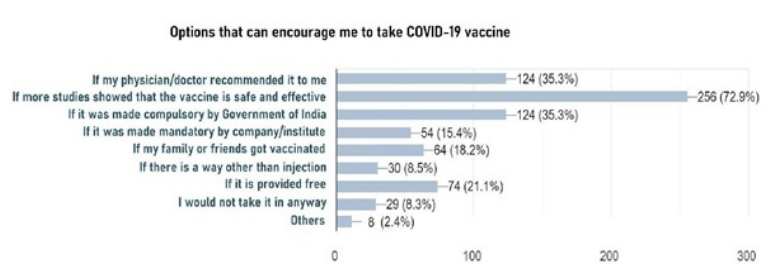

Figure 4 Response of participants regarding the factors that can encourage them to take COVID-19 vaccination. Image from Sharun et al. COVID-19 Vaccine Acceptance: Beliefs and Barriers Associated with Vaccination Among the General Population in India. J Experiment Bio Agri Sci. 2020; 8:S210-8

A total of $72.9 \%$ of the participants mentioned that they would agree to vaccination if further studies confirm the safety and effectiveness of it. 35.3\% of the participants responded that their physician's recommendation might motivate them to get vaccinated. Some of the participants mentioned that the Government of India $12(35.3 \%)$ or their company/institute (15.4\%) should make vaccination mandatory. Additionally, $21.1 \%$ want a vaccine to be given free of cost, while $8.3 \%$ responded that they would not take the COVID-19 vaccination under any circumstances. [13]

\begin{tabular}{|c|c|c|}
\hline Group & & Views on COVID-19 vaccine \\
\hline \multirow{4}{*}{ Doctors } & Knowledge & Updated with the current research and government policies \\
\hline & Attitude & Positive outlook towards the vaccine \\
\hline & Perception & $\begin{array}{l}\text { Acceptability of COVID-19 vaccine amongst doctors will impact overall acceptability in } \\
\text { the general population }\end{array}$ \\
\hline & Concerns & $\begin{array}{l}\text { The gap in Information, Education and Communication for the acceptance of the } \\
\text { vaccine }\end{array}$ \\
\hline \multirow{4}{*}{$\begin{array}{l}\text { Subject } \\
\text { experts }\end{array}$} & Knowledge & Updated with the current research and government policies \\
\hline & Attitude & Mixed views, some willing to promote it while others being skeptical \\
\hline & Perception & Vaccine should be taken and/or promoted for correct reasons \\
\hline & Concerns & $\begin{array}{l}\text { Misinformation spread from social media about the vaccine and limited direction } \\
\text { given by the government }\end{array}$ \\
\hline \multirow{4}{*}{ Paramedics } & Knowledge & Information obtained from scientific data and news channels \\
\hline & Attitude & Mixed views, some supporting while others being skeptical \\
\hline & Perception & $\begin{array}{l}\text { Vaccine has been launched without adequate data as compared to other vaccines } \\
\text { being used worldwide }\end{array}$ \\
\hline & Concerns & Safety and efficacy of the vaccine for elderly and comorbid individuals \\
\hline \multirow{4}{*}{$\begin{array}{l}\text { Other hospital } \\
\text { staff }\end{array}$} & Knowledge & Obtained knowledge/information from doctors and paramedics \\
\hline & Attitude & Positive outlook \\
\hline & Perception & Vaccine is crucial to curb COVID-19 spread \\
\hline & Concerns & $\begin{array}{l}\text { Initiation of the process of vaccination for other family members especially pregnant } \\
\text { women, children and elderly; rumours spread on social media }\end{array}$ \\
\hline \multirow{4}{*}{$\begin{array}{l}\text { Middle-aged } \\
\text { and elderly }\end{array}$} & Knowledge & Obtained information from news channels and by discussing it with family and friends \\
\hline & Attitude & Many were skeptical \\
\hline & Perception & Found the vaccine important but doubtful for its safe use on them \\
\hline & Concerns & Concerned about the safety and efficacy; authenticity; rumours \\
\hline \multirow{4}{*}{$\begin{array}{l}\text { Working } \\
\text { professionals }\end{array}$} & Knowledge & Limited interest and knowledge \\
\hline & Attitude & Casual attitude towards the vaccine \\
\hline & Perception & People of this age do not need any vaccine \\
\hline & Concerns & About the efficacy; rapid development and approval of the vaccine \\
\hline \multirow{4}{*}{ Young adults } & Knowledge & $\begin{array}{l}\text { Lacked complete information as were dependent on google and news applications } \\
\text { installed on their phones }\end{array}$ \\
\hline & Attitude & Mixed outlooks, some were positive while others being skeptical \\
\hline & Perception & The vaccine should be made available to college students and teachers \\
\hline & Concerns & Unforeseen future effects of the vaccine \\
\hline \multirow{4}{*}{ Housewives } & Knowledge & $\begin{array}{l}\text { Obtained through news channels, youtube videos and discussions with neighbours, } \\
\text { family and relatives }\end{array}$ \\
\hline & Attitude & Most were skeptical \\
\hline & Perception & Introduction of the vaccine has made people careless \\
\hline & Concerns & About the safety and efficacy, use for children and elderly, price of the vaccine \\
\hline
\end{tabular}




\section{Group-Wise Analysis of Vaccine Acceptance}

Table 1 Group-wise analysis of views on COVID-19 vaccine (Self Made) Data Source: A. Kumari, P. Ranjan, S. Chopra et al. What Indians Think of the COVID-19 vaccine: A qualitative study comprising focus group discussions and thematic analysis. Diabetes \& Metabolic Syndrome: Clinical Res. \& Reviews 15 (2021) 679-682 [14].

There are numerous sources of information regarding COVID-19 vaccination, from scientific research to social media, and as a result, there is misinformation which leads to confusion. Some groups, such as housewives, young adults, etc., rely on social media or discussion with family members to get informed about the vaccine. These groups are more hesitant to take the COVID-19 vaccine, which can be due to a lack of reliable sources of information. However, hospital groups had better knowledge of the COVID-19 vaccine. It can be seen that the level of knowledge and information is not related to vaccine acceptance as both, hospital and general population groups, had mixed opinions, but reliable information sources can help in improving acceptance. Positive attitude towards vaccination was linked with trust in the safety and efficacy of vaccines, free access to the vaccine, feeling of duty towards eliminating COVID-19 virus, perceived limited or no risk in vaccination, better and more reliable sources of information and easy availability of the COVID-19 vaccine. The concerns that contributed to negative attitude were decreased active COVID-19 cases in the country, preference for natural immunity (vaccine not required), misinformation and conspiracies, fear of vaccine side-effects, concerns regarding the genuineness of the vaccine etc. [14]

\section{Methods to Improve Vaccine Acceptance}

Public willingness to accept the COVID-19 vaccine depends on the knowledge and sentiment regarding the vaccine. The accelerated development of the vaccine might have contributed to the rise of concerns and negative attitudes among the general public. Vaccine awareness and its acceptance depend on various socio-demographic factors. The biggest barrier towards vaccine acceptance is the concern regarding mild or serious side effects following vaccination.

The following are few ways in which vaccine acceptance can be improved:
- Development of reliable information and communication channels to make people aware of the vaccine benefits and availability through media.

- The government and local authorities should take the initiative through social media engagement and alleviate concerns regarding vaccine-related issues like safety and efficacy while providing correct information. They should also try to resolve community-based problems like false beliefs, lack of knowledge and mistrust.

- Sharing correct information by local and community leaders, celebrities, and public figures can motivate the general public to accept the vaccine soon.

- Promoting confidence and supporting the healthcare workers in their decision to get vaccinated and also to recommend it to their patients. As we saw in the above case studies, a doctor's recommendation is an important factor that can help people get rid of their misinformation and concerns regarding vaccination.

- If more studies confirm the safety and efficacy of the COVID-19 vaccine, especially for the newer strains of the coronavirus, and if such information is made available via a centralized source of information, it may resolve many vaccine-related concerns among the public.

- Interventional education campaigns targeted at those who are at a higher risk for vaccine hesitancy and more susceptible to misinformation are essential.

- More studies aimed at understanding factors contributing to vaccine hesitancy are also necessary to help public health policymakers make more efficient strategies to implement the public vaccination program. [15]

\section{Conclusion}

COVID-19 pandemic has caused a huge loss of life and livelihood across the world. The development of vaccines is a ray of hope towards the end of this crisis. Vaccine hesitancy poses a serious threat to achieving global health as more time allows the virus to undergo mutations. It is possible that the vaccines may not be effective on all mutations of the virus in the future. It, therefore, becomes necessary to overcome this barrier of vaccine hesitancy. 
The vaccine acceptance rates vary across different countries according to various socio-demographic, community and psychological factors. The main concerns preventing the public from getting vaccinated are associated with short-term or longterm side effects, safety and necessity of the vaccines. Beliefs such as vaccination is not required for certain age groups are also dangerous. Better acceptance rates can be achieved if more rigorous studies prove that vaccines are safe and effective. Along with that, targeted awareness strategies can be useful in resolving vaccine-related concerns among the public. The sooner people accept the COVID-19 vaccine, the sooner the world will come out of the pandemic.

\section{Reference}

01. Singhal T. A Review of Coronavirus Disease2019 (COVID-19). Indian J Pediatr. 2020 Apr;87(4)281-286. doi: 10.1007/s12098-02003263-6 [Crossref][PubMed][Google Scholar]

02. Al-Mohaithef M, Padhi BK. Determinants of COVID-19 Vaccine Acceptance in Saudi Arabia- A Web-Based National Survey. J Multidiscip Healthc. 2020 Nov 20;13;1657-1663. doi: 10.2147/JMDH.S276771 [Crossref][PubMed][Google Scholar]

03. Larson HJ, Clarke RM, Jarrett C, Eckersberger E, Levine Z, Schulz WS, Paterson P. Measuring trust in vaccination- A systematic review. Hum Vaccin Immunother. 2018 Jul 3;14(7)1599-1609. doi: $10.1080 / 21645515.2018 .1459252 \quad$ [Crossref] [PubMed][Google Scholar]

04. Xiao X, Wong RM. Vaccine hesitancy and perceived behavioral control- A meta-analysis. Vaccine. 2020 Jul 14;38(33)5131-5138. doi: 10.1016/j.vaccine.2020.04.076 [Crossref][PubMed] [Google Scholar]

05. Gidengil CA, Parker AM, Zikmund-Fisher BJ. Trends in risk perceptions and vaccination intentions- a longitudinal study of the first year of the H1N1 pandemic. Am J Public Health. 2012 Apr;102(4)672-9. doi: 10.2105/AJPH.2011.300407 [Crossref][PubMed][Google Scholar]

06. Setbon $M$, Raude J. Factors in vaccination intention against the pandemic influenza $A / H 1 N 1$. Eur J Public Health. 2010 Oct;20(5)490-4. doi: 10.1093/eurpub/ckq054 [Crossref][PubMed][Google Scholar]
07. Halpin C, Reid B. Attitudes and beliefs of healthcare workers about influenza vaccination. Nurs Older People. 2019 Mar 22;31(2)32-39. doi: 10.7748/nop.2019.e1154 [Crossref][PubMed] [Google Scholar]

08. Sallam M. COVID-19 Vaccine Hesitancy Worldwide- A Concise Systematic Review of Vaccine Acceptance Rates. Vaccines (Basel). 2021 Feb 16;9(2)160. doi: 10.3390/vaccines 9020160 [Crossref][PubMed][Google Scholar]

09. Islam MS, Kamal AM, Kabir A, Southern DL, Khan SH, Hasan SMM, et al. COVID-19 vaccine rumors and conspiracy theories- The need for cognitive inoculation against misinformation to improve vaccine adherence. PLoS One. 2021 May 12;16(5)e0251605. doi: 10.1371/journal.pone.0251605 [Crossref][PubMed] [Google Scholar]

10. World health organization. How are vaccines developed?. Available from: https://www. who. int/news-room/feature-stories/detail/how-are-

vaccines-developed [Crossref][PubMed][Google Scholar]

11. Centers for disease control and prevention. How vaccines work. Available from: https://www. cdc. gov/coronavirus/2019-ncov/vaccines/different-

vaccines/how-they-work.html [Crossref][PubMed] [Google Scholar]

12. Sharun K, Dhama K. India's role in COVID-19 vaccine diplomacy. J Travel Med. 2021 Apr 16;taab064. doi: 10.1093/jtm/taab064 [Crossref] [PubMed][Google Scholar]

13. Sharun K, Rahman CKF, Haritha CV, Jose B, Tiwari R, Kuldeep D. Covid-19 vaccine acceptanceBeliefs and barriers associated with vaccination among the general population in india. J Exp Biol Agric Sci. 2020 Oct 31;8(Spl-1- SARS-CoV-2)S210S218. doi:10.18006/2020.8(Spl-1-SARS-CoV2).S210.S218 [Crossref][PubMed][Google Scholar]

14. Kumari $A$, Ranjan $P$, Chopra $S$, Kaur D, Kaur $T$, Kalanidhi KB, et al. What Indians Think of the COVID-19 vaccine- A qualitative study comprising focus group discussions and thematic analysis. Diabetes Metab Syndr. 2021 Mar 26;15(3)679-682. doi: 10.1016/j.dsx.2021.03.021 [Crossref][PubMed] [Google Scholar] 
15. Schoch-Spana $M$, Brunson EK, Long $R$, Ruth $A$, Ravi SJ, Trotochaud $M$, et al. The public's role in COVID-19 vaccination- Human-centered recommendations to enhance pandemic vaccine awareness, access, and acceptance in the United States. Vaccine. 2020 Oct 29;S0264410X(20)31368-2. doi: 10.1016/j.vaccine.2020.10.059 [Crossref][PubMed] [Google Scholar] 